\title{
К теории адсорбции на графеноподобных соединениях
}

\author{
() С.Ю. Давыдов \\ Физико-технический институт им. А.Ф. Иофре Российской академии наук, \\ Санкт-Петербург, Россия \\ Санкт-Петербургский национальный исследовательский университет \\ информационных технологий, механики и оптики, \\ Санкт-Петербург, Россия \\ E-mail: Sergei_Davydov@mail.ru
}

(Получена 24 мая 2016 г. Принята к печати 20 июня 2016 г.)

На основании предложенной ранее модели электронного спектра бинарных графеноподобных соединений типа $\mathrm{A}_{N} \mathrm{~B}_{8-N}$ построена теория адсорбции, позволяющая выявить роль положения уровня адатома, величины константы взаимодействия адатом-подложка и ширины щели, присущей в свободном состоянии графеноподобному соединению с гетерополярными связями, в формировании электронной структуры адатома. Рассмотрены случаи свободных и эпитаксиальных графеноподобных соединений на поверхности металла. В случае свободных графеноподобных соединений анализ показал, что при больших и промежуточных значениях константы связи адатом-графеноподобное соединение основной вклад в число заполнения адатома $n_{a}$ дают локальные состояния, тогда как с уменьшением константы связи возрастает вклад валентной зоны графеноподобного соединения. Основной особенностью эпитаксиального графеноподобного соединения на металле является отсутствие щели и, как следствие, вклада локальных состояний адатома в $n_{a}$. Оценки показали, что изменения констант связей адатом-подложка и графеноподобное соединение-металл влияют на величину $n_{a}$ практически одинаковым образом. При этом зависимость $n_{a}$ от ширины щели графеноподобного соединения с качественной точки зрения критичной не является. Кратко обсуждается адсорбция на структуре графеноподобное соединение-полупроводник.

DOI: 10.21883/FTP.2017.02.44110.8336

\section{1. Введение}

После появления графеновой тематики [1,2], представляющей чрезвычайный интерес как с фундаментальной $[3,4]$, так и с прикладной $[5,6]$ точки зрения, начался поиск других двумерных (2D) структур, обладающих по возможности преимуществами однослойного графена и лишенных его недостатков [7]. К последним можно отнести отсутствие щели (запрещенной зоны) в электронном спектре идеального однолистного графена, что ограничивает возможности его использования в приборных структурах. Для создания такой щели приходится прибегать к так называемой функционализации, в качестве которой могут выступать легирование (допирование), адсорбция, подложка (в случае эпитаксиального графена), неоднородная деформация и т.д.

В полупроводниковой электронике общеизвестна огромная роль трехмерных (3D) бинарных соединений co структурой цинковой обманки типа $\mathrm{A}_{N} \mathrm{~B}_{8-N}$, где A(B) - атом металла (неметалла). В работе [8] теоретически исследовалась возможность существования 2D аналогов таких соединений. Исследование 27 таких материалов, каждому из которых было позволено образовывать три 2D решетки (см. рис. 1 в [8]), показало, что устойчивыми являются 38 структур, причем 12 из них могут сосуществовать в двух фазах. Гексагональная структура представляет собой обычную плоскую графеноподобную решетку, характеризуемую $\sigma$-связями $s p^{2}$-орбиталей. По результатам расчетов [8] такой решеткой обладают $\mathrm{BN}, \mathrm{BP}, \mathrm{BAs}, \mathrm{BSb}, \mathrm{GaN}$,
$\mathrm{InN}, \mathrm{ZnO}$ и BeO. Тетрагональная структура, присущая $\mathrm{AlN}, \mathrm{AlAs}, \mathrm{AlSb}, \mathrm{BeSe}$ и BeTe, является слоистой, в которой каждый атом $\mathrm{A}(\mathrm{B})$ окружен эквидистантно расположенными четырьмя атомами $\mathrm{B}(\mathrm{A})$ с длиной связи A-B порядка 2-2.5 А. Измятая структура характерна для $\mathrm{AlP}, \mathrm{GaP}, \mathrm{GaAs}, \mathrm{GaSb}, \mathrm{InP}, \mathrm{InAs}, \mathrm{InSb}, \mathrm{ZnS}, \mathrm{ZnSe}$, $\mathrm{ZnTe}, \mathrm{CdS}, \mathrm{CdSe}, \mathrm{CdTe}$ и BeS. При этом измятость слоя, определяемая расстоянием между верхними и нижними атомами (см. рис. 1 в [8]), значительна, $\sim(1-4) \AA$, а длины связей $\mathrm{A}(\mathrm{B})$ с тремя ближайшими соседями $\mathrm{B}(\mathrm{A})$ не одинаковы, так что приписать этой структуре чистые случаи $s p^{2}$ - или $s p^{3}$-гибридизации невозможно.

Модельному подходу к теоретическому описанию структур $\mathrm{A}_{N} \mathrm{~B}_{8-N}$, которые в дальнейшем будем именовать графеноподобными соединениями (ГПС), посвящены работы $[9,10]$, где наряду со свободными слоями рассмотрены слои эпитаксиальные, так как именно таковыми являются слои ван-дер-ваальсовых, или вертикальных, структур [11], перспективных для приборного использования. В настоящей работе мы рассмотрим задачу об адсорбции на таких соединениях, свободных и сформированных на металле и (лишь схематично) на полупроводнике. При этом мы будем использовать низкоэнергетическое приближение для электронного спектра свободного ГПС $[3,9,10]$, что, вообще говоря, в задаче об адсорбции может приводить к определенным неточностям [12]. Мы, однако, идем на подобное упрощение, так как цель настоящей работы состоит в том, чтобы сформулировать схему решения задачи об адсорбции на ГПС и оценить, как положение уровня адатома, 
величина константы взаимодействия адатом-подложка и ширина щели, присущей в свободном состоянии ГПС с гетерополярными связями (когда $\mathrm{A} \neq \mathrm{B}$ ), влияют на адсорбционные характеристики адатома.

\section{2. Свободные графеноподобные соединения}

Из самых общих соображений [13] функция Грина $G_{a}(\omega)$ для атома, адсорбированного на эпитаксиальном графене, может быть записана в виде

$$
G_{a}^{-1}(\omega)=\omega-\varepsilon_{a}-\Lambda_{a}(\omega)+i \Gamma_{a}(\omega) .
$$

Здесь $\omega-$ энергетическая переменная, $\varepsilon_{a}-$ энергия одноэлектронного уровня адатома; функция уширения квазиуровня адатома есть

$$
\Gamma_{a}(\omega)=\pi V_{a / \mathrm{sub}}^{2} \rho_{\mathrm{sub}}(\omega),
$$

где $\rho_{\text {sub }}(\omega)$ - плотность состояний (ПС) подложки, $V_{a / \text { sub }}$ - матричный элемент взаимодействия адатом-подложка; функция сдвига квазиуровня есть

$$
\Lambda_{a}(\omega)=\frac{1}{\pi} P \int_{-\infty}^{\infty} \frac{\Gamma_{a}\left(\omega^{\prime}\right) d \omega^{\prime}}{\omega-\omega^{\prime}}
$$

где $P$ - символ главного значения. ПС на адатоме $\rho_{a}(\omega)$, отвечающая функции Грина $(1)$, имеет вид

$$
\rho_{a}(\omega)=\frac{1}{\pi} \frac{\Gamma_{a}(\omega)}{\left[\omega-\varepsilon_{a}-\Lambda(\omega)\right]^{2}+\Gamma_{a}^{2}(\omega)},
$$

а число заполнения $n_{a}$ уровня $\varepsilon_{a}$ адатома при нулевой температуре равно

$$
n_{a}=\int_{-\infty}^{E_{\mathrm{F}}} \rho_{a}(\omega) d \omega
$$

где $E_{\mathrm{F}}-$ уровень Ферми подложки. Таким образом, определив ПС подложки $\rho_{\text {sub }}(\omega)$, можно вычислить характеристики адсорбированного состояния. Отметим, что здесь и далее мы рассматриваем одноэлектронный (или однодырочный) атом, т.е. атом, который в силу внутриатомного кулоновского отталкивания может содержать на внешней орбите лишь один электрон (одну дырку) [13].

В работе [9] в низкоэнергетическом приближении показано, что ПС свободного бинарного ГПС АВ дается выражением

$$
\rho_{\mathrm{AB}}(\Omega)= \begin{cases}\frac{2|\Omega|}{\xi^{2}}, & \sqrt{\xi^{2}+\Delta^{2}} \geq|\Omega| \geq|\Delta|, \\ 0, & |\Omega|<|\Delta|, \quad|\Omega|>\sqrt{\xi^{2}+\Delta^{2}},\end{cases}
$$

где $\Omega=\omega-\bar{\varepsilon}, \bar{\varepsilon}=\left(\varepsilon_{a}+\varepsilon_{b}\right) / 2, \Delta=\left(\varepsilon_{a}-\varepsilon_{b}\right) / 2, \varepsilon_{a(b)}-$ энергия $p$-орбитали атома $\mathrm{A}(\mathrm{B}), \xi=\sqrt{2 \pi \sqrt{3}} t, t-$ энергия перехода между $p_{z}$-орбиталями ближайших атомов А и $\mathrm{B}$, нуль энергии помещен в центр щели, а в бесщелевом случае переходит в точку Дирака. Полагая $\rho_{\text {sub в }}(2)$ равным $\rho_{\text {АВ }}$, получим

$$
\Lambda_{a}(\Omega)=\frac{2 V_{a / \mathrm{sub}}^{2}}{\xi^{2}} \Omega \ln \left|\frac{\Omega^{2}-\Delta^{2}}{\Omega^{2}-\Delta^{2}-\xi^{2}}\right|
$$

При $\Delta=0$ выражение (7) переходит в формулу (6) работы [12] для свободного графена. Некоторые аналитические свойства функции $\Lambda_{a}(\Omega)$ приведены в Приложении, п. 1.

Для дальнейшего анализа введем константу взаимодействия $\alpha=2 V_{a / \mathrm{sub}}^{2} / \xi^{2}$ и перейдем к относительным единицам $x=\Omega / \xi, \eta_{a}=\varepsilon_{a} / \xi, \bar{e}=\bar{\varepsilon} / \xi, \delta=|\Delta| / \xi$. Тогда безразмерные функция уширения и сдвига есть $\gamma_{a}(x)=\Gamma_{a}(x) / \xi$ и $\lambda_{a}(x)=\Lambda_{a}(x) / \xi$. Функции $\lambda_{a}(x)$ изображены на рис. 1 совместно с безразмерной ПС $f_{\mathrm{AB}}(x)=\rho_{\mathrm{AB}}(x) \xi$ свободного ГПС. Обращает на себя внимание качественное различие функций $\lambda_{a}(x)$ в отсутствие (рис. $1, a)$ и при наличии (рис. $1, b)$ щели в спектре.

ПС на адатоме (4) в приведенном виде можно теперь записать как

$$
\bar{\rho}_{a}(x) \equiv \rho_{a}(\omega) \xi=\frac{\alpha f_{\mathrm{AB}}(x)}{\left[x+\eta-\lambda_{a}(x)\right]^{2}+\left[\pi \alpha f_{\mathrm{AB}}(x)\right]^{2}},
$$

где $\eta=\left(\bar{\varepsilon}-\varepsilon_{a}\right) / \xi=\bar{e}-\eta_{a} \quad$ и $\quad \lambda_{a}(x)=\alpha x \ln \mid\left(x^{2}-\delta^{2}\right) /$ $\left(x^{2}-\delta^{2}-1\right) \mid$. Плотность состояний атома, адсорбированного на однослойном графене, исследовалась достаточно подробно (см., например, $[13,14]^{1}$ ), так что в настоящей работе мы сосредоточимся на ГПС с ненулевой щелью.

Результаты расчета функций $\bar{\rho}_{a}(x)$ представлены на рис. 2 для энергий, соответствующих сплошному спектру ГПС, когда $\sqrt{1+\delta^{2}} \geq|x| \geq \delta$. Из рисунка следует, что при выбранных для расчета типичных параметрах задачи, $\eta=0, \delta=0.25$ (рис. $2, a)$ и $\delta=0.75$ (рис. $2, b$ ), константы взаимодействия $\alpha=1$ величина ПС на адатоме $\bar{\rho}_{a}(x) \sim 0.1$. Действительно, в соответствии с (8), максимальное значение $\bar{\rho}_{a}\left(x_{r}\right) \sim \alpha \pi^{-2} \bar{f}^{-1}\left(x_{r}^{-}\right)$, где $x_{r}^{-}$- резонансное состояние в области валентной зоны (см. далее). Так как $f\left(x_{r}^{-}\right) \sim 1$ (см. рис. 2) и $\alpha=1$, то $\bar{\rho}_{a}\left(x_{r}^{-}\right) \sim \pi^{-2} \approx 0.1$. В случае сильной связи адатом-подложка $(\alpha \gg 1)$ ПС $\bar{\rho}_{a}(x)$ становится исчезающе малой, тогда как при слабой связи $(\alpha \ll 1)$ ПС $\bar{\rho}_{a}(x)$ может достигать значительной величины. Какихлибо качественных различий $\bar{\rho}_{a}(x)$ для случаев $\delta=0.25$ и $\delta=0.75$ не наблюдается.

Перейдем теперь к локальным состояниям, безразмерные энергии которых $x_{l}$ определяются корнями уравнения

$$
x+\eta-\lambda_{a}(x)=0
$$

в области энергий вне сплошного спектра ГПС $(|x|<\delta$, $\left.|x|>\sqrt{1+\delta^{2}}\right)$, где функция $f_{\mathrm{AB}}(x) \equiv x$. Графический

\footnotetext{
${ }^{1}$ Следует подчеркнуть, что в $[13,14]$ использовалось не низкоэнергетическое приближение, а так называемая М-модель ПС однолистного графена. Основные результаты, однако, не зависят сколь-либо критически от модели, а связаны с линейной зависимостью ПС от энергии в области точки Дирака и обращению в нуль в самой этой точке.
} 

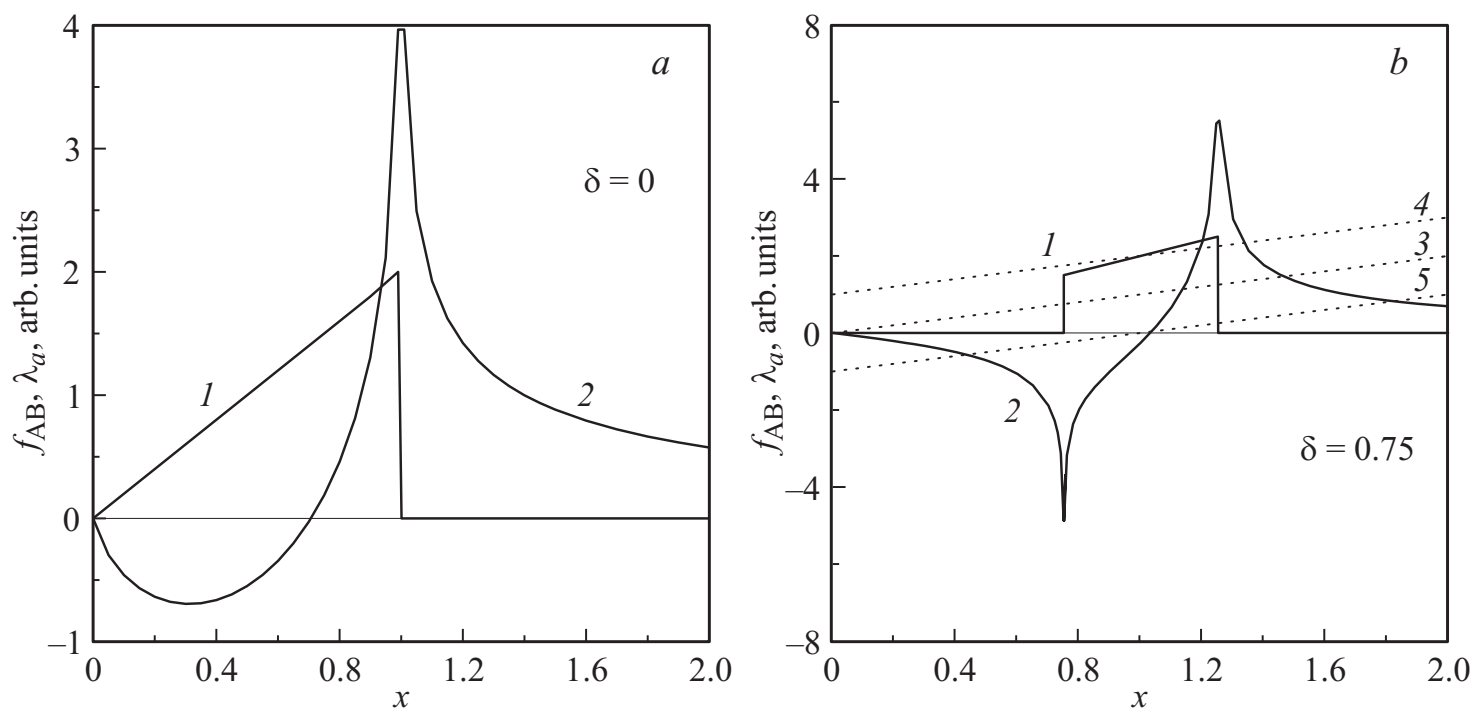

Рис. 1. Зависимости приведенной плотности состояний ГПС $f_{\text {Ав }}(1)$ и функции сдвига квазиуровня адатома $\lambda_{a}(2)$ от безразмерной энергии $x=\omega / \xi$ при константе взаимодействия $\alpha-1$ и приведенной полуширине щели $\delta=0(a), 0.75(b)$. Показаны только правые части функций $f_{\text {Ав }}$ и $\lambda_{a}$, так как первая из них является четной, а вторая нечетной. Расходимости функции $\lambda_{a}$, возникающие на границах сплошного спектра ГПС, заменены конечными пиками. $b$ : тонкими пунктирными линиями изображены функции $x+\eta$ при $\eta=0(3), 1(4)$ и $-1(5)$.
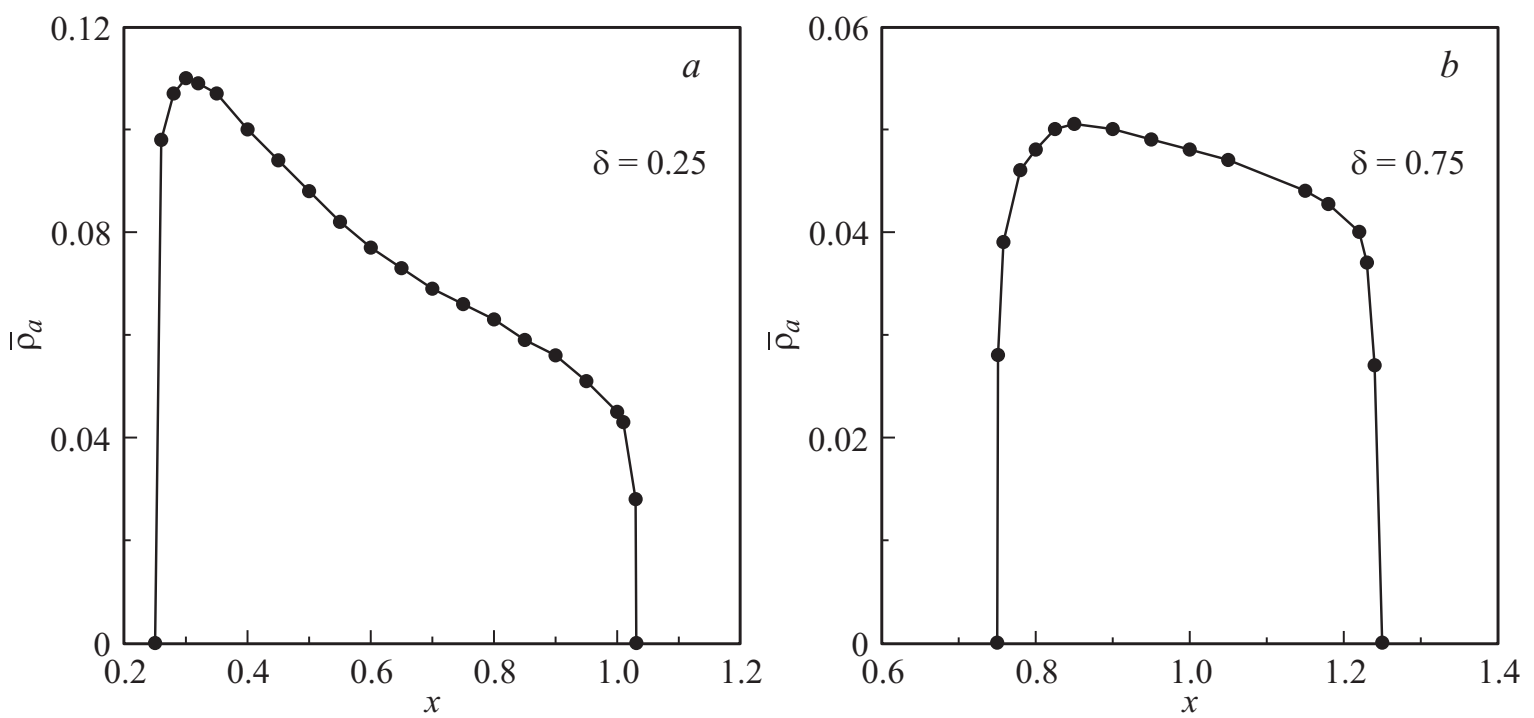

Рис. 2. Зависимости приведенных плотностей состояний на адатоме $\bar{\rho}_{a}$ от безразмерной энергии $x=\omega / \xi$ при $\alpha=1, \eta=0$, безразмерной полуширине щели $\delta=0.25(a), 0.75(b)$ для области энергии, отвечающей сплошному спектру ГПС. Показана только правая часть четной при $\eta=0$ функции $\bar{\rho}_{a}(x)$.

способ решения уравнения (9) при наличии щели в спектре представлен на рис. $1, b$ : точки пересечения пунктирных линий $x+\eta$ с приведенной функцией сдвига $\lambda_{a}(x)$ определяют значения уровней, наведенных адатомом. Легко сообразить, что имеются два резонансных уровня $x_{r}^{+}$и $x_{r}^{-}$, расположенных соответственно в областях зоны проводимости и валентной зоны. Имеются и три локальных уровня, два из которых, $x_{l}^{+}$и $x_{l}^{-}$, расположены соответственно выше потолка зоны проводимости и ниже дна валентной зоны, а третий локальный уровень, $x_{l 0}$, лежит в области щели. При $\alpha=1$ и $\eta=0$ легко показать, что $x_{l 0}=0$ и $x_{l}^{+}=\sqrt{\delta^{2}+e /(e-1)}$, где $e-$ основание натурального логарифма. Тогда $x_{l}^{+} \approx 1.28$ при $\delta=0.25$ и $x_{l}^{+} \approx 1.46$ при $\delta=0.75$.

Из рис. $1, b$ видно, что при $\eta=-1$ в системе возникает локальный уровень $x_{l 0}$, расположенный в верхней половине щели. Легко сообразить (см. (9)), что при $\eta=1$ в нижней половине щели имеется аналогичный зеркально расположенный локальный уровень. Расчет по формуле (9) для $\alpha=1$ и $\eta=1$ дает следующие резуль- 

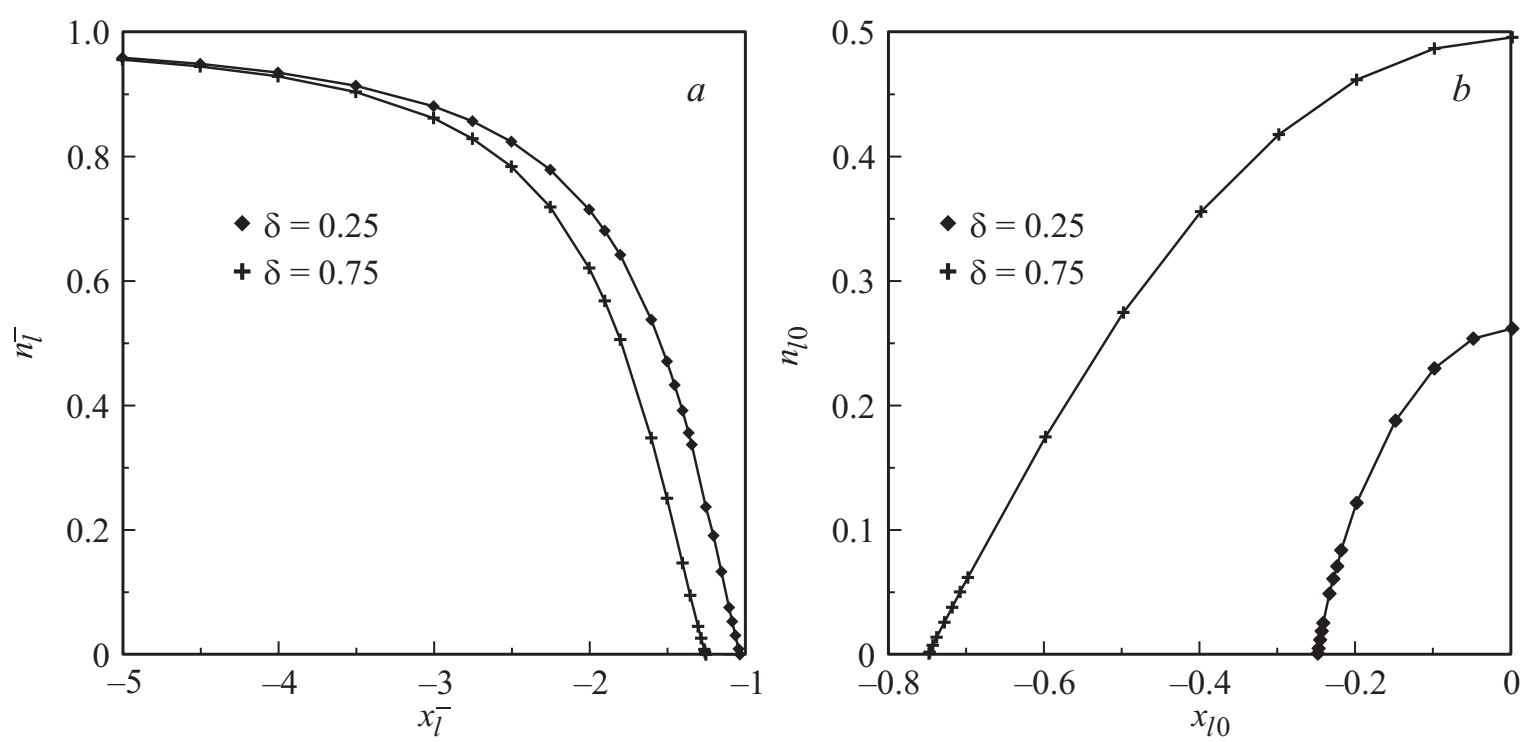

Рис. 3. Зависимости чисел заполнения $n_{l}^{-}(a)$ и $n_{l 0}(b)$ от безразмерных энергий локальных уровней $x_{l}^{-}$и $x_{l 0}$, лежащих соответственно под валентной зоной и в нижней части щели ГПС.

таты: при $\delta=0.25$ имеем $x_{10} \approx-0.20$; при $\delta=0.75$ получаем $x_{l 0} \approx-0.44$. Качественных различий для случаев $\delta=0.25$ и $\delta=0.75$ не наблюдается. Зависимость корней $x_{l}^{-}$и $x_{l}^{+}$для различных значений параметров задачи обсуждается в Приложении, п. 2.

Перейдем теперь к вычислению числа заполнения адатома $n_{a}$. Это число удобно представить в виде суммы [13], первое слагаемое в которой есть число заполнения $n_{\text {band }}$, представляющее собой вклад валентной зоны, равный

$$
n_{\mathrm{band}}=\int_{-\sqrt{1+\delta^{2}}}^{-\delta} \bar{\rho}_{a}(x) d x
$$

Для случаев, изображенных на рис. $2, n_{\text {band }} \approx 0.05$ при $\delta=0.25$ и $n_{\text {band }} \approx 0.02$ при $\delta=0.75$. Полезная качественная оценка значений $n_{\text {band }}$ приведена в Приложении, п. 3.

Последующие слагаемые $n_{\text {loc }}$ определяются локальными уровнями. Это, во-первых, вклад уровня $x_{l}^{-}$, лежащего ниже дна валентной зоны и поэтому всегда заполненного. Во-вторых, это вклад находящегося в щели локального уровня $x_{l 0}$ при условии, что он находится ниже приведенного уровня Ферми $\varepsilon_{\mathrm{F}}=E_{\mathrm{F}} / \xi$. Как известно [13], число заполнения $n_{\mathrm{loc}}$ локального состояния $x_{\text {loc }}$ дается выражением

$$
n_{\mathrm{loc}}=\left|1-\frac{d \lambda_{a}(x)}{d x}\right|_{x_{\mathrm{loc}}}^{-1},
$$

где $x_{\text {loc }}-$ приведенная энергия локального уровня. Выражение для производной $d \lambda_{a}(x) / d x$ дано в Приложении, п. 4 (см. (П3)). Для случая $\alpha=1$ и $\eta=0$ значения локальных вкладов уровней $x_{l}^{-} \approx-1.28$ $(\delta=0.25)$ и $x_{l}^{-} \approx-1.46 \quad(\delta=0.75)$, лежащих ниже дна валентной зоны ГПС, равны соответственно $n_{l}^{-} \approx 0.22$ и $n_{l}^{-} \approx 0.21$. Уровень $x_{l 0}$ дает вклад $n_{l 0}=\left[1+\alpha \ln \left(1+\delta^{2}\right) / \delta^{2}\right]^{-1}$, откуда при $\alpha=1$ получаем $n_{l 0} \approx 0.26(\delta=0.25)$ и $n_{l 0} \approx 0.49(\delta=0.75)$. Следовательно, $n_{\mathrm{loc}} \approx 0.48(\delta=0.25)$ и $n_{\mathrm{loc}} \approx 0.70(\delta=0.75)$, a полные числа заполнения адатома $n_{a}=n_{\text {band }}+n_{\text {loc }}$ равны соответственно $n_{a} \approx 0.53(\delta=0.25)$ и $n_{a} \approx 0.72$ $(\delta=0.75)$. Здесь мы предположили, что уровень Ферми $\varepsilon_{\mathrm{F}}=0^{+}$, т. е. смещен на бесконечно малую величину в верхнюю половину щели, так что локальный уровень $x_{l 0}=0$ является занятым.

В случае $\alpha=1$ и $\eta=1$ значения локальных вкладов уровней $x_{l 0} \approx-0.20(\delta=0.25)$ и $x_{l 0} \approx-0.44$ $(\delta=0.75)$, расположенных в нижней половине щели, соответственно равны $n_{l 0}^{-} \approx 0.20$ и $n_{l 0}^{-} \approx 0.33$. Общий вид зависимостей $n_{l}^{-}\left(x_{l}^{-}\right)$и $n_{l 0}\left(x_{l 0}\right)$ представлен на рис. 3, а некоторые частные случаи приведены в Приложении, п. 4. Вновь следует отметить отсутствие качественных различий для случаев $\delta=0.25$ и $\delta=0.75$.

Таким образом, вклад локальных состояний $n_{\mathrm{loc}}=n_{l}^{-}$ $+\vartheta\left(\varepsilon_{\mathrm{F}}-x_{l 0}\right) n_{l 0}$, где $\vartheta(z)-$ функция Хэвисайда, равная 1 при $z>0$ и 0 при $z \leq 0$, является превалирующим по сравнению с вкладом валентной зоны $n_{\text {band }}$ для промежуточной и сильной связи адатом-ГПС $(\alpha \geq 1)$. Такая ситуация характерна, например, для адсорбции атомов водорода и галогенов на графене [14]. С уменьшением константы $\alpha$ вклад зонных состояний возрастает, а локальных уменьшается. При слабой связи адатом-ГПС вклады $n_{\text {band }}$ могут стать превалирующими, как это имеет место при адсорбции атомов щелочных металлов на графене [14]. Действительно, для оценки величины константы $\alpha$ можно использовать простые выражения $[13,14]$. Так, например, для адатомов с внешней 

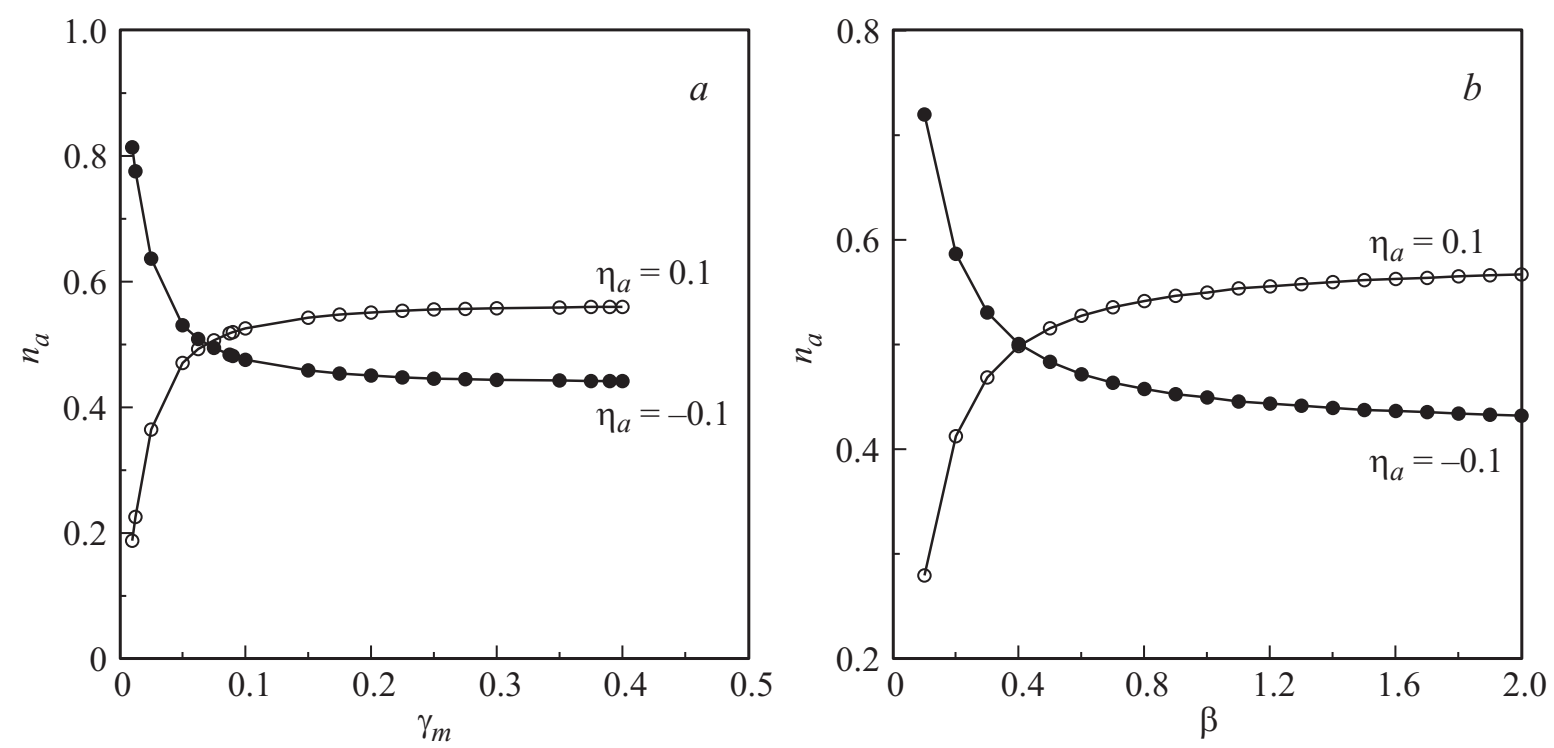

Рис. 4. Зависимости числа заполнения адатома $n_{a}$ от констант связи $\gamma_{m}$, описывающей взаимодействие ГПС с металлической подложкой $(a)$, и $\beta$, отвечающей взаимодействию адатома с системой ГПС/металл $(b)$ при $\delta=0.25, \eta_{a}= \pm 0.1, \beta=1(a)$, $\gamma_{m}=0.2(b)$.

орбиталью $|s\rangle_{a}$, спаривающейся с $\left|p_{z}\right\rangle$-орбиталью ГПС, получаем $\alpha \approx 21.5 / d^{4} t^{2}$, где энергия перехода $t$ задается в эВ, а длина адсорбционной связи $d-$ в А. Полагая $t \approx 3$ эВ и принимая для атомарного водорода $d \approx 1 \AA$, получим $\alpha \approx 2$. Для адатомов с внешней орбиталью $|p\rangle_{a}$, спаривающейся с $\left|p_{z}\right\rangle$-орбиталью ГПС, получаем $\alpha \approx 52.7 / d^{4} t^{2}$. Так как для адатомов фтора $d \approx 2 \AA$, имеем $\alpha \approx 1$. Для щелочных металлов $d>2 \AA$, так что $\alpha<1$.

В принципе для расчета числа заполнения $n_{\text {band }}$ при адсорбции атома на свободном ГПС можно было бы построить аналитическую модель, аналогичную предложенной в $[13,14]$ М-модели адсорбции на графене, использовав при этом параметры ГПС, приведенные в [9]. Однако в настоящее время отсутствуют не только данные эксперимента, но и соответствующие результаты расчетов других авторов. Поэтому мы сочли такую задачу преждевременной.

\section{3. Эпитаксиальные графеноподобные соединения}

Начнем с рассмотрения плоских 2D слоев ГПС, сформированных на металле. Как показано в работе [9], металлическая подложка, ПС которой описывается простейшей моделью $\rho_{\text {sub }}(\omega)=\rho_{\text {met }}=$ const, сглаживает все характерные особенности ПС свободного ГПС. При этом главный эффект воздействия металлической подложки заключается в исчезновении щели ГПС как области запрещенных состояний (см. формулу (13) и рис. 3 в [9]). В соответствии с [9] и Приложением, п. 5, безразмерная ПС плоского слоя эпитаксиального ГПС на металле $\bar{\rho}_{\mathrm{AB}}^{\mathrm{met}}(x)$ в нормировке настоящей работы дается формулой (П6). В [9] также показано, что при $\delta^{2} \ll 1$ и $\gamma_{m}^{2} \ll 1$ при $x^{2} \ll 1$ эта ПС может быть сведена к квадратичной зависимости вида $\bar{\rho}_{\mathrm{AB}}^{\mathrm{met}}(x) \approx A+C x^{2}$, где константы $A$ и $C$ определяются выражениями (П7) и (П8) Приложения, п. 5.

Аналогично (2), полуширина квазиуровня атома, адсорбированного на ГПС, сформированном на металле, есть

$$
\Gamma_{a}^{\mathrm{AB} / \mathrm{met}}(\omega)=\pi\left(V_{a}^{\mathrm{AB} / \mathrm{met}}\right)^{2} \rho_{\mathrm{AB}}^{\mathrm{met}}(\omega),
$$

или, в приведенном виде, $\gamma_{a m}=\Gamma_{a}^{\mathrm{AB} / \mathrm{met}} / \xi$ (здесь $V_{a}^{\mathrm{AB} / \mathrm{met}}$ - матричный элемент взаимодействия адатома со структурой ГПС-металл). Функция сдвига квазиуровня $\Lambda_{a}^{\mathrm{AB} / \mathrm{met}}(\omega)$ задается формулой, аналогичной (3). Если положить константу связи адатома с ГПС на металле равной $\beta=\left(V_{a}^{\mathrm{AB} / \mathrm{met}}\right)^{2} / \xi^{2}$, то $\lambda_{a m}(x) \equiv \Lambda_{a}^{\mathrm{AB} / \mathrm{met}} / \xi=\beta\left(A+C x^{2}\right) \ln |(x-\delta) /(x+\delta)|$. Coответствующая приведенная ПС на адатоме дается попрежнему формулой (8), но с заменой $\alpha$ на $\beta, f_{\mathrm{AB}}(x)$ на $\bar{\rho}_{\mathrm{AB}}^{\mathrm{met}}(x)$ и $\lambda_{a}(x)$ на $\lambda_{a m}(x)$.

Для оценки перехода заряда между адатомом и подложкой будем для простоты считать, что приведенный уровень Ферми системы ГПС/металл $\varepsilon_{\mathrm{F}}=0$, так что между металлом и ГПС перехода заряда не происходит [9]. Пусть далее $|\eta| \ll 1$. Аппроксимируем ПС на адатоме лоренцевым контуром вида

$$
\bar{\rho}_{a}(x)=\frac{\beta A}{\left(x-x_{0}\right)^{2}+(\pi \beta A)^{2}},
$$

где мы положили $\bar{\varepsilon}=0$, так что $\eta=-\eta_{a}$, $x_{0} \approx \eta_{a}+\lambda_{a m}\left(\eta_{a}\right)$. Расчеты показывают, что при $\delta^{2} \ll 1$ и $\gamma_{m}^{2} \ll 1$ имеем $A \approx C$, так что в области $x^{2} \ll 1$ можно положить $\bar{\rho}_{\mathrm{AB}}^{\mathrm{met}}(x) \approx A$. Тогда число заполнения 
адатома $n_{a}$ приближенно равно

$$
n_{a} \approx \frac{1}{\pi} \operatorname{arcctg} \frac{x_{0}}{\pi \beta A}
$$

Результаты расчетов зависимостей $n_{a}\left(\gamma_{m}\right)$ и $n_{a}(\beta)$ представлены на рис. 4, $a$ и $b$ соответственно. Обращает на себя внимание не только качественное, но и количественное соответствие зависимостей $n_{a}\left(\gamma_{m}\right)$ и $n_{a}(\beta)$. Этот результат свидетельствует о том, что обе силовые константы влияют на заполнение орбитали адатома одинаковым образом. Более детально этот вопрос рассмотрен в Приложении, п. 5. Там же показано, что зависимость $n_{a}$ от величины $\delta$ можно считать слабой.

Спад значений $n_{a}$ с ростом констант связи $\gamma_{m}$ и $\beta$ для отрицательных значений приведенной энергии квазиуровня адатома объясняется положительным значением функции сдвига $\lambda_{a m}\left(\eta_{a}\right)$ : действительно, сдвигаясь в сторону положительных энергий, квазиуровень адатома опустошается. При $\eta_{a}>0$ имеет место обратный эффект: с увеличением $\gamma_{m}$ и $\beta$ заполнение адатома нарастает, что связано со сдвигом квазиуровня адатома в сторону отрицательных энергий. Из выражения (14) следует также, что при замене $\eta_{a}$ на $-\eta_{a}$ число заполнения $n_{a}$ переходит в $1-n_{a}$, что и отображает рис. 4 . Выход зависимостей $n_{a}\left(\gamma_{m}\right)$ и $n_{a}(\beta)$ на насыщение с ростом $\gamma_{m}$ и $\beta$ объясняется в Приложении, п. 5. Таким образом, вследствие отсутствия щели в ГПС на металле локальные уровни адатомов отсутствуют и вклад в число заполнения $n_{a}$ полностью определяется состояниями сплошного спектра, занимающего в рамках использованной модели все энергетическое пространство.

Перейдем теперь к адсорбции на плоских 2D эпитаксиальных слоях ГПС, сформированных на полупроводнике. Возможные типы гетеропереходов, образуемых контактом ГПС с полупроводниковой подложкой, рассмотрены в работе [10]. При этом в зависимости от соотношения ширин щели ГПС $2 \delta$ и запрещенной зоны подложки $E_{g}$, их взаиморасположения на шкале энергии и, главное, от константы связи ГПС-полупроводник имеет место большое разнообразие электронных структур, которые при определенных соотношениях параметров могут обладать целым набором подзон запрещенных состояний. Общая задача об адсорбции на такой структуре формулируется, однако, просто. Действительно, аналогично (12), можно записать функцию уширения квазиуровня в виде $\Gamma_{a}^{\mathrm{AB} / \mathrm{sc}}(\omega)=\pi\left(V_{a}^{\mathrm{AB} / \mathrm{sc}}\right)^{2} \rho_{\mathrm{AB}}^{\mathrm{sc}}(\omega)$, где $V_{a}^{\mathrm{AB} / \mathrm{sc}}-$ матричный элемент взаимодействия адатома со структурой ГПС/полупроводник, ПС которой $\rho_{\mathrm{AB}}^{\mathrm{sc}}(\omega)$ приведена в [10]. Подставляя $\Gamma_{a}^{\mathrm{AB} / \mathrm{sc}}(\omega)$ в выражение (3), найдем функцию сдвига квазиуровня $\Lambda_{a}^{\mathrm{AB} / \mathrm{sc}}(\omega)$, определив тем самым ПС на адатоме вида (4). Интересно отметить, что в данном случае, как и в случае свободных ГПС, вклад в число заполнения адатома $n_{a}$ будут вносить как зонные (резонансные), $x_{r}$, так и локальные, $x_{\text {loc }}$, состояния. Отметим, что последние являются аналогами щелевых состояний $x_{l 0}$ при адсорбции атомов на свободном ГПС.

\section{4. Заключение}

Итак, в настоящей работе мы построили общую схему рассмотрения задачи об адсорбции одиночного атома для свободных и эпитаксиальных плоских 2D слоев соединений $\mathrm{A}_{N} \mathrm{~B}_{8-N}$. Численные расчеты и полуколичественные оценки, выполненные для свободных ГПС и ГПС, сформированных на металле, позволили сделать ряд заключений о роли положения атомного уровня, ширины запрещенной зоны ГПС и соответствующих констант связи в формировании электронной структуры (ПС и чисел заполнения) адатома. Что же касается эпитаксиальных слоев ГПС на полупроводнике, то тут возникает (благодаря многофакторности задачи) слишком много возможных вариантов. Более того, в отличие от 2D ГПС на металле, реальность существования которых можно считать достаточно серьезно теоретически обоснованной $[15,16]$, ГПС на полупроводниковой подложке являются пока структурами гипотетическими. Поэтому в данной работе мы не стали проводить оценки адсорбционных характеристик.

При адсорбции одиночного атома нам в принципе все равно, с каким именно атомом подложки, А или В, он связан, так как это обстоятельство отражается только на величине константы связи. Иное дело, когда встает задача о конечной концентрации адатомов. При этом адсорбция может изменить ширину щели ГПС в свободном состоянии (по аналогии с наведенной адсорбцией щели в графене), что, естественно, проявится и в случае эпитаксиального ГПС. Постановку подобной задачи мы также считаем преждевременной.

\section{Приложение}

1. Воспользовавшись формулой (7), легко показать, что при $\Omega \rightarrow 0$ функция сдвига $\Lambda_{a}(\Omega) \rightarrow 0$ как $\Omega \ln \left[\Delta^{2} /\left(\Delta^{2}+\xi^{2}\right)\right]$ для $\Delta \neq 0$ и как $\Omega \ln (|\Omega| / \xi)$ для $\Delta=0$. Две другие точки, где $\Lambda_{a}(\Omega)$ обращается в нуль, равны $\Omega_{0}^{ \pm}=\sqrt{\Delta^{2}+\xi^{2} / 2}$. При $\Omega_{1}^{ \pm}= \pm \Delta$ и $\Omega_{2}^{ \pm}= \pm \sqrt{\Delta^{2}+\xi^{2}}$ функция $\Lambda_{a}(\Omega)$ логарифмически расходится как $\Lambda_{a}(\Omega) \propto \Omega_{1}^{ \pm} \ln \left(\left|\Omega^{2}-\left(\Omega_{1}^{ \pm}\right)^{2}\right| / \xi^{2}\right)$ и $\Lambda_{a}(\Omega) \propto \Omega_{2}^{ \pm} \ln \left(\xi^{2} /\left|\Omega^{2}-\left(\Omega_{2}^{ \pm}\right)^{2}\right|\right)$ соответственно. При $|\Omega| \rightarrow \infty$ получаем $\Lambda_{a}(\Omega) \propto \xi^{2} / \Omega$. Все перечисленные особенности отражены на рис. 1 .

2. Обратимся к решению уравнения (9). Рассмотрим значения корней $x_{l}^{-}$и $x_{l}^{+}$, отвечающих состояниям, лежащим соответственно ниже дна валентной зоны и выше потолка зоны проводимости, т. е. при $x^{2}>\delta^{2}+1$. Пусть $|\eta| \gg 1$. Тогда в области $|x| \gg \sqrt{1+\delta^{2}}$ мы можем записать $\lambda_{a}(x) \approx \alpha / x$. В результате получаем $x_{l}^{ \pm}=\eta\left(1+\sqrt{1+4 \alpha^{2} / \eta^{2}}\right)$, где верхний индекс ,Плюс относится к $\eta>0$, а индекс „минус“ - к $\eta<0$. Отметим, что в рассмотренном пределе значения $x_{l}^{-}$и $x_{l}^{+}$не зависят от ширины щели $\delta$.

Пусть теперь $|\eta| \ll 1$. Предполагая, что уровни $x_{l}^{-}$и $x_{l}^{+}$лежат вблизи внешних границ сплошного спектра 
ГПС, положим $\lambda_{a}(x) \approx \alpha \sqrt{1+\delta^{2}} \ln \left[1 /\left(x^{2}-\delta^{2}-1\right)\right]$. Тогда $\left(x_{l}^{ \pm}\right)^{2} \approx 1+\delta^{2}+\exp \left(-\sqrt{1+\delta^{2}} / \alpha\right)$. С ростом ширины щели локальные уровни прижимаются к границам разрешенных зон. То же наблюдается при увеличении константы взаимодействия $\alpha$.

Перейдем теперь к локальным состояниям $x_{10}$, pacположенным в области щели. Пусть $|\eta| \gg 1$. Тогда ясно, что уровень $x_{l 0}$ будет лежать вблизи потолка валентной зоны при $\eta<0$ и вблизи дна зоны проводимости при $\eta>0$. Легко показать, что $x_{l 0}^{2} \approx 1+\delta^{2}$ $-\exp \left(-\sqrt{1+\delta^{2}} / \alpha\right)$. При $|\eta| \ll 1$ имеем $x+\eta \approx \alpha x$ $\times \ln \left[\delta^{2} /\left(1+\delta^{2}\right)\right]$, откуда $x_{l 0} \approx-\eta /\left\{1+\alpha \ln \left[\left(1+\delta^{2}\right) / \delta^{2}\right]\right\}$.

3. С учетом (6) представим ПС на адатоме (8) для области энергий, соответствующей валентной зоне ГПС, в виде

$$
\bar{\rho}_{a}(x) \approx \frac{1}{\pi} \frac{2 \pi \alpha\left|x_{r}^{-}\right|}{\left(x-x_{r}^{-}\right)^{2}+\left(2 \pi \alpha x_{r}^{-}\right)^{2}} .
$$

Тогда в соответствии с (10) получим

$$
n_{\mathrm{band}} \approx \frac{1}{\pi}\left(\operatorname{arctg} \frac{x_{r}^{-}+\sqrt{1+\delta^{2}}}{2 \pi \alpha\left|x_{r}^{-}\right|}-\operatorname{arctg} \frac{x_{r}^{-}+\delta}{2 \pi \alpha\left|x_{r}^{-}\right|}\right) .
$$

Максимальное значение зонного вклада $n_{\text {band }}$ имеет порядок $(2 / \pi) \operatorname{arctg}(1 / 2 \pi \alpha)$, при $\alpha \gg 1$ имеем $n_{\text {band }} \sim\left(\pi^{2} \alpha\right)^{-1}$. Эта же оценка справедлива и при $\alpha \sim 1$ (см. текст статьи). При $\alpha \ll 1$ получаем $n_{\text {band }} \sim 1$. Этот результат имеет простой физический смысл: при слабой связи с подложкой ГПС является квазисвободной структурой с одним электроном, заполняющим валентную зону.

4. Продифференцировав выражение (7), в приведенной форме получим

$$
\frac{d \lambda_{a}(x)}{d x}=\alpha \ln \left|\frac{\delta^{2}-x^{2}}{\delta^{2}+1-x^{2}}\right|-\frac{2 \alpha x^{2}}{\left(\delta^{2}-x^{2}\right)\left(\delta^{2}+1-x^{2}\right)} .
$$

Легко видеть, что на границах зон, т.е. при $x^{2} \rightarrow \delta^{2}$ и $x^{2} \rightarrow \delta^{2}+1$, функция $\left|d \lambda_{a}(x) / d x\right| \rightarrow \infty$, а при $|x| \rightarrow \infty$ получаем $d \lambda_{a}(x) / d x \rightarrow-\alpha / x^{2}$.

Рассмотрим вклады локальных состояний, лежащих ниже дна валентной зоны и выше потолка зоны проводимости. При $|\eta| \gg 1$ имеем в соответствии с п. 2 Приложения $x_{l}^{ \pm}=\eta\left(1+\sqrt{1+4 \alpha^{2} / \eta^{2}}\right)$,

$$
d \lambda_{a}(x) / d x \sim-\alpha / \eta^{2}\left(1+\sqrt{1+4 \alpha^{2} / \eta^{2}}\right)^{2},
$$

откуда в соответствии с (11) получаем

$$
n_{l}^{-} \sim\left[1+\alpha / \eta^{2}\left(1+\sqrt{1+4 \alpha^{2} / \eta^{2}}\right)^{2}\right]^{-1},
$$

откуда (при разумных значениях $\alpha$ ) имеем $n_{l}^{-} \sim 1$. Отметим, что при этом плотность состояний на адатоме в области валентной зоны $\bar{\rho}_{a}(x) \sim 2 \alpha\left|x_{r}\right| / \eta^{2} \ll 1$, так что $n_{\text {band }} \sim 0$.

При $|\eta| \ll 1$ в соответствии с п. 2 Приложения имеем $\left(x_{l}^{-}\right)^{2} \approx 1+\delta^{2}+\exp \left(-\sqrt{1+\delta^{2}} / \alpha\right)$, откуда, согласно (11), получаем $n_{l}^{-} \sim 0$.
Теперь обратимся к вкладам локальных состояний, расположенных в щели ГПС. Как показано в п. 2 Приложения, в пределе $|\eta| \gg 1$ имеем $x_{l 0}^{2} \approx 1+\delta^{2}-\exp \left(-\sqrt{1+\delta^{2}} / \alpha\right)$, так что $n_{l 0} \sim 0$. В пределе $|\eta| \ll 1$ имеем $n_{l 0} \sim\left\{1+\alpha \ln \left[\left(1+\delta^{2}\right) / \delta^{2}\right]\right\}^{-1}$. Здесь мы считали, что имеем дело с собственным образцом ГПС, уровень Ферми которого $\varepsilon_{\mathrm{F}}=0$.

5. В работе [9] за единицу энергии принималось значение интеграла перехода $t$, тогда как в настоящей работе используется параметр $\xi$. В соответствии с этой заменой плотность состояний эпитаксиального ГПС на металлической подложке имеет вид

$$
\begin{aligned}
\bar{\rho}_{\mathrm{AB}}^{\mathrm{met}}(x) \equiv & \rho_{\mathrm{AB}}^{\mathrm{met}}(x) \xi=\frac{\gamma_{m}}{\pi} \ln \frac{\left|1+b^{\prime}+c^{\prime}\right|}{c^{\prime}} \\
& +\frac{2 x}{\pi}\left(\operatorname{arctg} \frac{2+b^{\prime}}{4 \gamma_{m} x}-\operatorname{arctg} \frac{b^{\prime}}{4 \gamma_{m} x}\right) .
\end{aligned}
$$

Здесь $\quad b^{\prime}=-2\left(x^{2}-\delta^{2}-\gamma_{m}^{2}\right), \quad c^{\prime}=\left(x^{2}-\delta^{2}\right)^{2}+\gamma_{m}^{2}$ $\times\left(\gamma_{m}^{2}+2 \delta^{2}+2 x^{2}\right) \quad$ и $\quad \gamma_{m}=\Gamma_{m} / \xi$, где $\quad \Gamma_{m}=\pi V_{m}^{2} \rho_{\text {met }}$ и $V_{m}-$ матричный элемент взаимодействия ГПС c металлической подложкой (см. подробнее в [9]). В предположении, что $\delta^{2} \ll 1$ и $\gamma_{m}^{2} \ll 1$, выражение (П6) при $x^{2} \ll 1$ может быть сведено к

$$
\bar{\rho}_{\mathrm{AB}}^{\mathrm{met}}(x) \approx \bar{\rho}_{\mathrm{AB}}^{\mathrm{met}}(0)+\frac{2 \gamma_{m}}{\pi} \frac{3 \delta^{2}+\gamma_{m}^{2}}{\delta^{2}+\gamma_{m}^{2}} x^{2},
$$

где

$$
\bar{\rho}_{\mathrm{AB}}^{\mathrm{met}}(0)=\frac{2 \gamma_{m}}{\pi} \ln \frac{1}{\delta^{2}+\gamma_{m}^{2}} .
$$

Отметим, что в тексте статьи коэффициент при $x^{2}$ во втором слагаемом выражения (П7) обозначен как $C$, $A=\bar{\rho}_{\mathrm{AB}}^{\operatorname{met}}(0)$.

Перейдем теперь к анализу формулы (15) и зависимостей $n_{a}\left(\gamma_{m}\right), n_{a}(\beta)$, представленных на рис. 4 . В области малых энергий $\lambda_{a m}(x) \approx-2 \beta A x$. Тогда, учитывая, что $A \propto \gamma_{m}$ (логарифмический множитель влияет слабо), получаем: значения сдвига квазиуровня адатома $\lambda_{a m}(x)$ и его полуширины $\pi \beta A$ пропорциональны произведению $\beta \gamma_{m}$. Именно в этом и заключается причина подобия зависимостей $n_{a}\left(\gamma_{m}\right)$ и $n_{a}(\beta)$. Отметим также, что величина коэффициента $A=\bar{\rho}_{\mathrm{AB}}^{\text {met }}(0)$ зависит от полуширины щели $\delta$ логарифмически, т. е. сравнительно слабо.

При значениях силовых констант $\gamma_{m}$ и $\beta$, когда сдвиг квазиуровня $\left|\lambda_{a m}\left(\eta_{a}\right)\right|$ начинает существенно превышать его начальную энергию $\left|\eta_{a}\right|$, функции $n_{a}\left(\gamma_{m}\right)$ и $n_{a}(\beta)$ выходят на насыщение. Действительно, при $x^{2} \ll 1$ получаем, что $n_{a} \rightarrow 0.5+\pi^{-1} \operatorname{arctg}\left(2 \eta_{a} / \pi\right)$.

\section{Список литературы}

[1] А.К. Гейм. УФН, 181, 1284 (2011).

[2] К.С. Новосёлов. УФН, 181, 1299 (2011).

[3] A.H. Castro Neto, F. Guinea, N.M.R. Peres, K.S. Novoselov, A.K. Geim. Rev. Mod. Phys., 81, 109 (2009).

[4] M.I. Katsnelson. Graphene. Carbon in Two Dimensions (UK, Cambridge Univ. Press, 2012). 
[5] Graphene Nanoelectronics. Metrology, Synthesis, Properties and Applications, ed. by H. Raza (Springer-Verlag, BerlinHeidelberg, 2012).

[6] П.Б. Сорокин, Л.А. Чернозатонский. УФН, 183, 113 (2013).

[7] A.K. Geim, I.V. Grigorieva. Nature, 499, 419 (2013).

[8] C.-J. Tong, H. Zhang, Y.-N. Zhang, H. Liu, L.-M. Liu. J. Mater. Chem. A, 2, 17971 (2014).

[9] С.Ю. Давыдов. ФТТ, 58, 779 (2016).

[10] С.Ю. Давыдов. ФТТ, 58, 1182 (2016).

[11] И.В. Антонова. ФТП, 50, 67 (2016).

[12] С.Ю. Давыдов, О.В. Посредник. ФТТ, 57, 1654 (2015).

[13] С.Ю. Давыдов. Теория адсорбиии: метод модельных гамильтонианов (СПб., Изд-во СПбГЭТУ „ЛЭТИ“, 2013). twirpx.com/file/1596114/

[14] С.Ю. Давыдов, Г.И. Сабирова. Письма ЖТФ, 37 (11), 51 (2011).

[15] H.L. Zhuang, A.K. Singh, R.G. Hennig. Phys. Rev. B, 87, 165415 (2013).

[16] A.K. Singh, H.L. Zhuang, R.G. Hennig. Phys. Rev. B, 89, 245431 (2014).

Редактор Л.В. Шаронова

\section{To the theory of adsorption \\ on graphene-like compounds}

S.Yu. Davydov

loffe Institute,

194021 St. Petersburg, Russia

St. Petersburg National Research University

of Information Technologies, Mechanics and Optics,

197101 St. Petersburg, Russia

Abstract On the basis of the earlier proposed model for the electron spectra of $\mathrm{A}_{N} \mathrm{~B}_{8-N}$ graphene-like compounds (GLC), adsorption theory is put forward. This theory permits to clarify the roles of adatom level position, adatom-substrate coupling constant and the GLC gap. Both free-standing GLC and epitaxial GLC formed on metal substrates are considered. For the freestanding GLC analysis shows that for the strong and intermediate adatom-GLC interaction the main contribution to the adatom's occupation number $n_{a}$ is given by the local states while with the decrease of this interaction the valance band contribution increases. The main feature of the GLC on metals is absence of the gap and, hence, zero local states contributions to $n_{a}$. The estimations demonstrate that the effects of the adatom-substrate and GLC-metal coupling constants on the $n_{a}$ value are practically the same. Also note that the width of the GLC gap plays noncritical role in the value of $n_{a}$. Adsorption of the GLC on semiconductor substrate is also briefly discussed. 